\title{
Ethics in health promotion and prevention of disease
}

\author{
Raanan Gillon Imperial College, St Mary's Hospital Medical School and King's College, London University
}

In this issue of the journal Dr Petr Skrabanek argues that health promotion - and especially experiments in health promotion - should be as subject to ethical scrutiny as any other aspect of health care - and offers evidence and arguments for concluding that at present it and they are not (1). The issues he raises deserve meticulous consideration, yet such is the contemporary enthusiasm for health promotion that they are all too easily brushed aside, especially perhaps by health care workers anxious to respond to the modern scorn for treatment of illness and disease when prevention is - of course - so much better.

In brief, Dr Skrabanek's arguments are that any intervention by doctors to promote people's health should produce net benefit for the populations served, and that all individuals offered such interventions should adequately understand the pros and cons for themselves as well as for others. But many health promotion interventions are introduced without adequate evidence of producing net benefit for the populations served by them, and without offering the individuals intervened-upon adequate information either about the pros and cons of the interventions for themselves or for others. He cites even such widely accepted health-promotion interventions as cholesterol-reducing diets in women, and screening for hypertension, cervical cancer and breast cancer, whether by mammography or self-examination, as examples where the benefits and or their extent are questioned and the potential harms underrated. His proposal is that all health promotion interventions that have not been thoroughly evaluated should be classified as medical research on populations 'population experiments' as he puts it - and should be subject to the same ethical assessment as are clinical trials. To this end he proposes the establishment of a forum representing public, legal and medical perspectives 'to identify the ethical problems posed by new developments in preventive medicine and health promotion'.

What objections might face the claim that health promotion interventions should be subject to the same degree of ethical scrutiny as any other medical interventions? The most intuitively appealing objection is, perhaps, that health promotion is so obviously a good and worthwhile activity that it is absurd to require ethical scrutiny. A second objection might be that it is interfering, meddlesome and unjustified for the State, or the medical profession as an institution, to seek to intervene in the freeinterchange between doctor (or other health cares professional) and patient or client. A third might bethat it is a waste of scarce health care resources.

So far as the first objection is concerned there is of course a way of interpreting it such that any 2 intervention that is not beneficial is not classed aș health promotion - thus it becomes analytically true that health promotion is necessarily beneficial. BSte that simply highlights the need to distinguish betwe proposed interventions that are and are not beneficiHealth promotion activities may always or generally intended to be beneficial - the same may well be true ofō other more straightforwardly 'therapeutic' medicalo interventions. One of the purposes of contemporary $\varrho$ ethical scrutiny is to subject such claims to assessment $\overrightarrow{{ }_{0}}$ by independent scrutineers. Nor is such assessment 3 simple. It requires specification of the nature of the benefits intended and anticipated, their probability, and the beneficiaries; moreover since to do anything to anyone is likely to involve some risk of harming the person, the harms too must be specified, as well as their probabilities and their anticipated victims.

Thus the apparently straightforward claim that 0 health promotion is obviously beneficial needs scrutiny not only to see whether as a matter of empirical fact it음 is true for the particular health promotion intervention $\square$ (and Dr Skrabanek surely gives sufficient reason to require that such claims are not simply accepted on $N$ trust), but also to ascertain who are the intended beneficiaries and who might be harmed, as well as the probabilities of such harms and benefits occurring. All $\omega$ this is now generally accepted for the introduction of new therapies. There seems no good reason to absolve health promotion interventions from the same need for $\Phi$ scrutiny - some will doubtless prove beneficial, but ${ }^{+}$ their claims need to be demonstrated. Until such ${ }^{\circ}$ benefits are demonstrated their introduction surely requires cautious evaluation, and subjects affected $\stackrel{?}{\odot}$

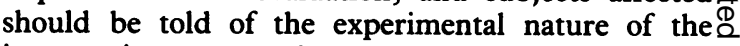
interventions proposed.

What about the second counterargument, that ethical scrutiny of health promotion interventions 
would require unjustified interference with the free interchange between doctor and patient? If the previous counterargument can be interpreted to be grounded in beneficence and non-maleficence, and an assumption that doctors should seek to provide net benefit-over-harm for their patients, this one can be seen as stemming from the requirement to respect autonomy - doctors and patients are autonomous agents and should be free to come to their own arrangements without third party interference. At first sight it is an attractive argument - what business is it of anyone else if a doctor advises a patient to have a cervical smear so as to detect and deal with any harmful abnormalities and the patient decides to accept? However, the argument leaves out of consideration a vital feature of the doctor-patient relationship, namely that the underlying assumption - unless explicitly negated - behind any normal medical intervenion is that it is intended and likely to provide net benefit-overharm for the patient intervened on. If those conditions are not met, then unless the patient is told that they are not met, to advise an intervention may be seen as overriding the patient's autonomy, for the patient properly assumes, unless informed to the contrary, that the conditions are met, and acts on that assumption - the conditions are part of the grounding for the patient's deciding to trust the doctor's advice.

But unless the intervention has been adequately assessed neither the doctor nor the patient can adequately know about the harms and benefits that may result, and their probabilities, and thus cannot be sure about the likelihood of the patient deriving net benefit over harm. Two conclusions seem to follow. First, that health promotion interventions need adequate assessment of their harms and benefits, as above. Second that pending such assessment, if a doctor advises the intervention without telling the patient that the harms and benefits have not been adequately assessed - that the intervention is essentially experimental - he is in effect deceiving the patient, given, as above, that patients normally properly assume that doctors' advice is based not only on an intention to benefit them but also on adequate reasons for believing that there is a reasonable likelihood of doing so. Such deceit prevents the patient from deliberating adequately on the doctor's plans and thus prevents him or her from making an autonomous decision - it treats the patient, in Kantian terminology, as a mere means and not as an end.

The third argument - that ethical scrutiny of health promotion interventions is a waste of scarce resources - may be seen as an argument in distributive justice. Again it may sound plausible if (a) it is assumed that all health promotion interventions are both beneficial and not harmful and (b) that the interventions themselves have no adverse effect on scarce resources. The two assumptions are often combined into a single adage 'prevention is better than cure' and the second expressed by the proverb 'a stitch in time saves nine'. But the question of whether or not particular health promotion interventions produce net benefit over harm is open to doubt as shown above, and provision of reliable answers to such questions is part of the ethical scrutiny proposed by Dr Skrabanek. Whether or not a health promotion intervention will save or increase the burden on resources will depend on the particular case. A campaign by GPs opportunistically to advise their patients against smoking may well save more resources than it costs, and may well prove to have a very low cost per life 'saved', or per 'QALY' (2). But other health promotion/disease prevention interventions may be very expensive - indeed, according to one estimate, the cost of saving lives by the British system of cervical cytology screening is between $£ 270,000$ and $£ 285,000$ per life saved, (with 40,000 smears and 200 excision biopsies needed for each one of those 'averted deaths') (3). Since part of the purpose of ethical analysis is to question costs and benefits the argument that it would waste scarce resources seems implausible.

There is another ethical problem in the realm of distributive justice - notably that in a more or less fixed health budget as more is spent on health promotion/ disease prevention less must inevitably be spent on the actual treatment of sick people. Now it is an obvious fallacy that health promotion/disease prevention will reduce the need for treatment of illness and disease - we will all get sick, ultimately sick enough to die. (As Dr Skrabanek says, the saving of nine stitches by one may be true in sewing but it isn't generally true in health care). It may be that as a society we would be ready to trade treatment of actual illnesses for postponement of anticipated ones but that itself is one of the ethical issues in distributive justice that health promotion/ disease prevention raises; for undoubtedly disease and death are part of the human lot - we can promote health, we can prevent some diseases and postpone others, and we can sometimes postpone death. The more we do so within a fixed health budget the less we shall have to spend on those who become ill.

The case for ethical scrutiny of health promotion/ disease prevention interventions and programmes seems a powerful one. It remains a further question whether such ethical scrutiny should be carried out by some form of ethics committee. However, as $\mathrm{Dr}$ Skrabanek argues, if the introduction of new medications is deemed to require scrutiny by an ethics committee it is difficult to see why at least new health promotion interventions should not also require such scrutiny. The whole issue seems important enough for the attentions of the putative national bioethics commission that the Nuffield Foundation is currently considering establishing.

\section{References}

(1) Skrabanek P. Why is preventive medicine exempted from ethical constraints? Fournal of medical ethics 1990 ; 16:187190.

(2) Teeling Smith G. Measurement and management in the NHS. London: Office of Health Economics, 1989: 14.

(3) Charny M C, Farrow S C, Roberts C J. The cost of saving a life through cervical cytology screening: implications for health policy. Health policy 1987; 7:345-359. 